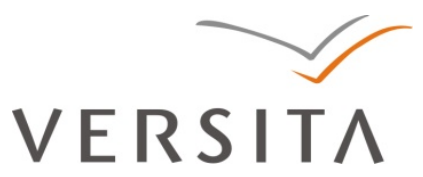

BULGARIAN ACADEMY OF SCIENCES

CYBERNETICS AND INFORMATION TECHNOLOGIES • Volume 13, Special Issue

Sofia $\bullet 2013$

Print ISSN: 1311-9702; Online ISSN: 1314-4081

DOI: 10.2478/cait-2013-0034

\title{
The Regional Medical Business Process Optimization Based on Cloud Computing Medical Resources Sharing Environment
}

\author{
A. Bei Xi, B. Qiuli Qin, C. Lei Huang \\ School of Economics and Management, Beijing Jiaotong University, Beijing, China \\ Emails:112120664@bjtu.edu.cn 2qlqin@bjtu.edu.cn 3lhuang@bjtu.edu.cn
}

\begin{abstract}
The paper elaborates some basic concepts, for example, cloud computing, medical resources sharing, business process optimization, and then points out the problems in the development process of regional health informatization. However, there are many good characteristics and advantages in cloud computing. If cloud computing can be used in the construction of regional health informatization, the problems like insufficient medical resources sharing, paying fees several times, repeated checkup, long hospitalization period, can be efficiently solved. So we put forward cloud computing service mode and research the architecture of regional medical collaborative cloud computing platform. It can realize medical resources sharing. We have a further study about business process optimization based on cloud computing medical resources sharing environment and find out that it can reduce repeated checkup, shorten hospitalization period, and so on.
\end{abstract}

Keywords: Information management, cloud computing, medical resource sharing, regional health informatization, regional medical collaborative cloud computing platform, business process optimization of regional medical treatment.

\section{Introduction}

At present the high quality medical resources are mostly concentrated in big cities and hospitals, while there is a lack of high-level medical resources in the local community health service institutions, which are geared to the ordinary people's 
needs. Many people travel long distances to see a doctor, which not only increases the economic burden, but also makes big hospitals overwhelmed. The results are that it is difficult to see a doctor and it is difficult to be accepted in a hospital. In the 21st century the development of cloud computing brought great influence in many industries only in a few years. If cloud computing can be used into health care industry, there will not only be a hope to solve the problems above mentioned, but also be a big promotion for the development of regional health informatization.

In [1] $\mathrm{H}$ a $\mathrm{n}$ has investigated that cloud computing is the latest computing model after distributed computing, parallel computing and grid computing . Cloud computing effectively integrates a variety of connected resources to achieve multilevel virtualization. Cloud computing is typically divided into three service offerings. The three service offerings are Software as a Service (SaaS), Platform as a Service (PaaS) and Infrastructure as a Service. In [2] Z h a o P. has investigated that the concept of medical resources sharing is that primary hospitals cooperate with superior hospitals which have high medical standards and advanced level of scientific research achievements based on the needs of patients and the aid of technology and equipment.

In [3] $\mathrm{L}$ a $\mathrm{i}, \mathrm{S}$ o $\mathrm{n}$ g have investigated that business process is very important and it is an information flow that reflects logistics process. Business process optimization is a strategy that develops, improves and optimizes the business processes to maintain competitive advantage. In [4] $\mathrm{H} \mathrm{o} \mathrm{w}$ a $\mathrm{r} d$, $\mathrm{P}$ e t e $\mathrm{r}$ have investigated that there are three phases in the process of execution business process optimization. Describe the business process: describe the enterprise business processes clearly through researching enterprises and collect relevant information. Analyze business process: analysis problems of the current process and find the causes. Propose and implement an optimization program: optimize the redundancy process and a relatively independent process to achieve the targets for improvement.

\section{The current situation of regional health informatization}

There are four stages in the development of medical informatization. In recent years, some powerful institutions began to explore regional health informatization which provides health management institutions, health care providers, patients and pharmaceutical products suppliers with a business and technology platform which delivers health industry data stored in a digital form to support the medical service. The process of realizing regional health informatization is not completely smooth. There are many problems. For example, medical resource misallocation, multilateral coordination difficulties, non-uniform standard and integration difficulties, insufficient capital investment.

\subsection{The current situation of a regional medical business process}

Diagnosis in community (Primary hospital).The patient should register and then he/she takes the registration form to wait to see a doctor. The doctor will make the judge whether patients need to check or not. If they do not need to check, the doctor 
will prescribe directly. If they need to check, they must pay fees first and then have a check. After several hours, they get the check results. After seeing the results, the doctor will make the judge whether they have the ability to solve the disease. If they can, they will prescribe. And then patients need to pay fees to get the medicine. If the patients need to be in a hospital, they should pay fees first and then have a treatment. When they recover, they will leave the primary hospital.

Upward referral. If the primary hospital doctors have no ability to solve the disease, they will transfer the patients to the superior hospital. If patients agree to referral, the doctor will fill out the upward referral list and then the business people will send it to the superior hospital. Doctors in the superior hospital will make a judge whether the patients need ambulatory treatment or hospital care. If they need ambulatory treatment, they need to have an outpatient treatment business process again and then leave. If they need hospital care, the doctor will write a hospital list. After paying fees, the patients will register to remain in hospital. They will be allocated a bed and an attending doctor. Then the patients need to have a check. After seeing the check results, the doctor will make a treatment plan. The doctor will give doctor's advice and the nurse will execute them.

Downward referral. When the conditions of the patients are improved after a period of treatment, the doctor thinks that the patients are in a stable condition and they can enter the stage of rehabilitation. If the patients agree to be transferred to the community hospital for rehabilitation, the business people will send the downward referral list written by the doctor, to the primary hospital. Doctors and nurses in the primary hospital will do rehabilitation treatment for the patients according to the superior hospital's doctors' orders until they recover and leave.

\subsection{The problems of regional medical business process}

In [5] $\mathrm{W}$ a $\mathrm{n}$ g, $\mathrm{S}$ u have investigated that although regional medical institutions have greatly improved the traditional business processes, there are still many problems.

1. Repeatedly providing basic information. When registering and checking in, the patients need to repeatedly provide their basic information. It is cumbersome and inefficient.

2. To pay for a few times. It is a waste of patients' time. In addition, the hospital needs a lot of charge windows, which results in wasting medical resources.

3. Checkup. It is inconvenient for the patients to carry their checkup results. After referral, the patients have to do the checkup repeatedly.

4. Doctor's orders. There may be something errors when saving doctor's orders and it is inconvenient for the patients to carry them.

5. Long period of hospitalization. After referral, the patients need to reregister, see a doctor, etc. The process is tedious, which may increase the hospitalization period. 


\section{The influence of cloud computing on regional health informatization}

We analyze the problems in the construction of regional medical informatization and know that cloud computing has a mass data storage, facilitates resource sharing, etc. If cloud computing can be used in medical industry, the problems faced in the regional medical informatization can be efficiently solved.

\subsection{Cloud computing service mode}

If cloud computing can be used in medical industry, we can put forward a cloud computing service mode, as shown in Fig. 1.

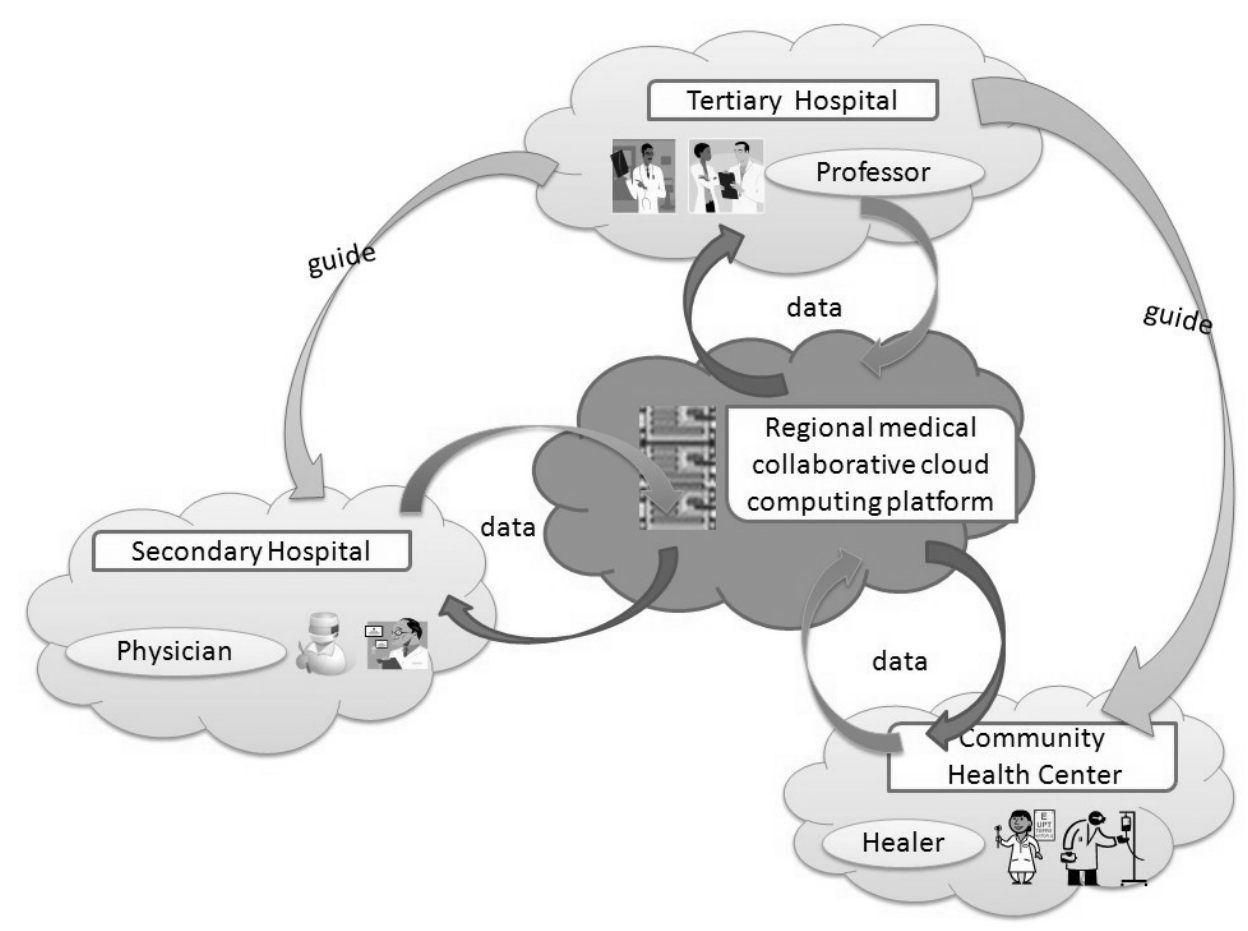

Fig.1. Cloud computing service mode

Regional medical collaborative cloud computing platform can be built based on the existing systems of a tertiary hospital, a second-class hospital, a community health center and so on. The platform has separate servers, databases, etc. According to the unified standard and related mechanisms, each side provides interfaces to connect with the cloud computing platform, transfers data to the platform and extracts data from the platform to realize the sharing of patients' information. After seeing the patients' information, the professors in tertiary hospitals can guide physicians in secondary hospitals and healers in community health centers. It can also achieve patient-centered medical services. For disease occurrence and development of the entire cycle, acquisition, organization, storage and application of the patient's disease related information, break the inter-agency, 
inter-professional information threshold to realize the exchange, sharing and integration of the patient information in a controlled state. So the patients can receive better medical services.

The architecture of a regional medical collaborative cloud computing platform is shown in Fig. 2.

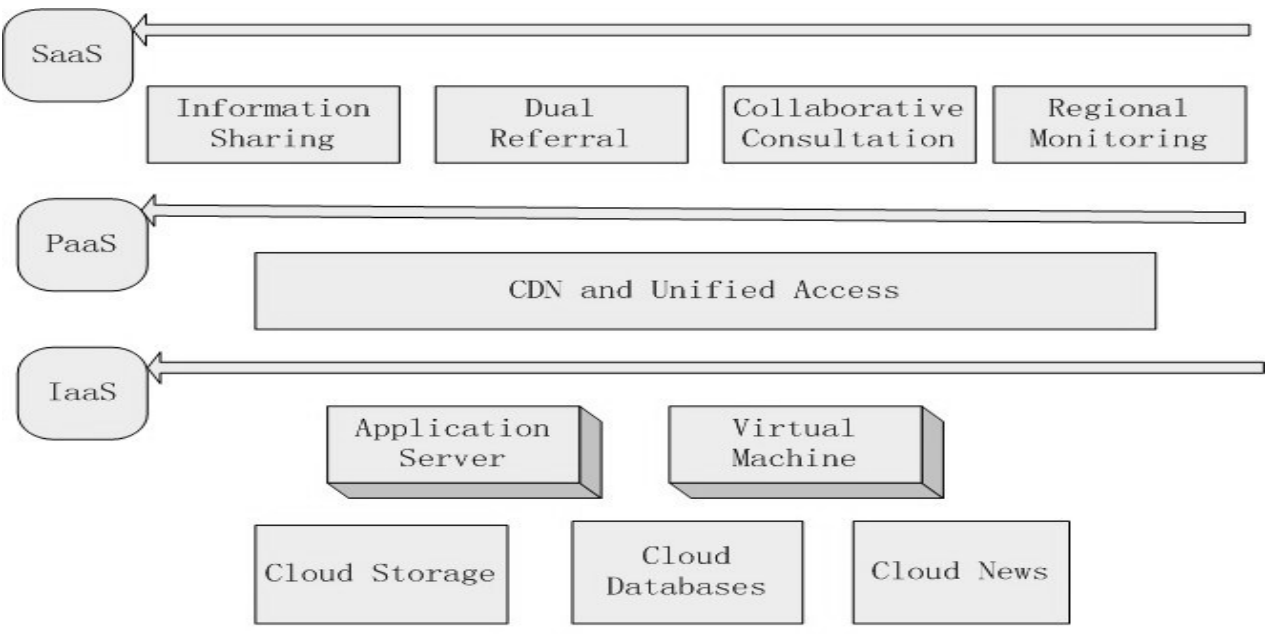

Fig. 2. The architecture of a regional medical collaborative cloud computing platform

In [6] T a n, L i u, S u n have investigated that cloud computing is typically divided into three service offerings. They are Software as a Service (SaaS), Platform as a Service (PaaS) and Infrastructure as a Service.

The IaaS layer provides cloud storage, cloud databases, cloud news and computing power which includes an application server and a virtual machine. The business process and data retention are processed in the cloud.

The SaaS layer provides optional software services, such as information sharing, dual referral, collaborative consultation, regional monitoring and so on. The users can selectively choose a part of the module and a function of the cloud computing platform.

The SaaS layer and the IaaS layer can be connected with the PaaS layer by the CDN (Content Delivery Network) and unified access. CDN can efficiently avoid the bottleneck of the data transmission speed and stability in Internet, which may affect the content transmission be faster and more stable.

The application architecture will be given in details in the next section.

3.2. The application architecture of regional medical collaborative cloud computing platform

In Fig. 3 the application architecture of a regional medical collaborative cloud computing platform is shown.

1. Platform hardware layer and database layer. The two layers correspond to the IaaS layer of cloud computing. They are the basis of the whole structure and can provide the basic support for the medical collaboration cloud computing 
platform. In [7] $\mathrm{Y}$ a $\mathrm{n}$ has investigated that these resources are integrated through virtualization technology and connected through Internet. The boundary of the server resources extends flexibly and loads evenly with the growth of more hospitals and patients.

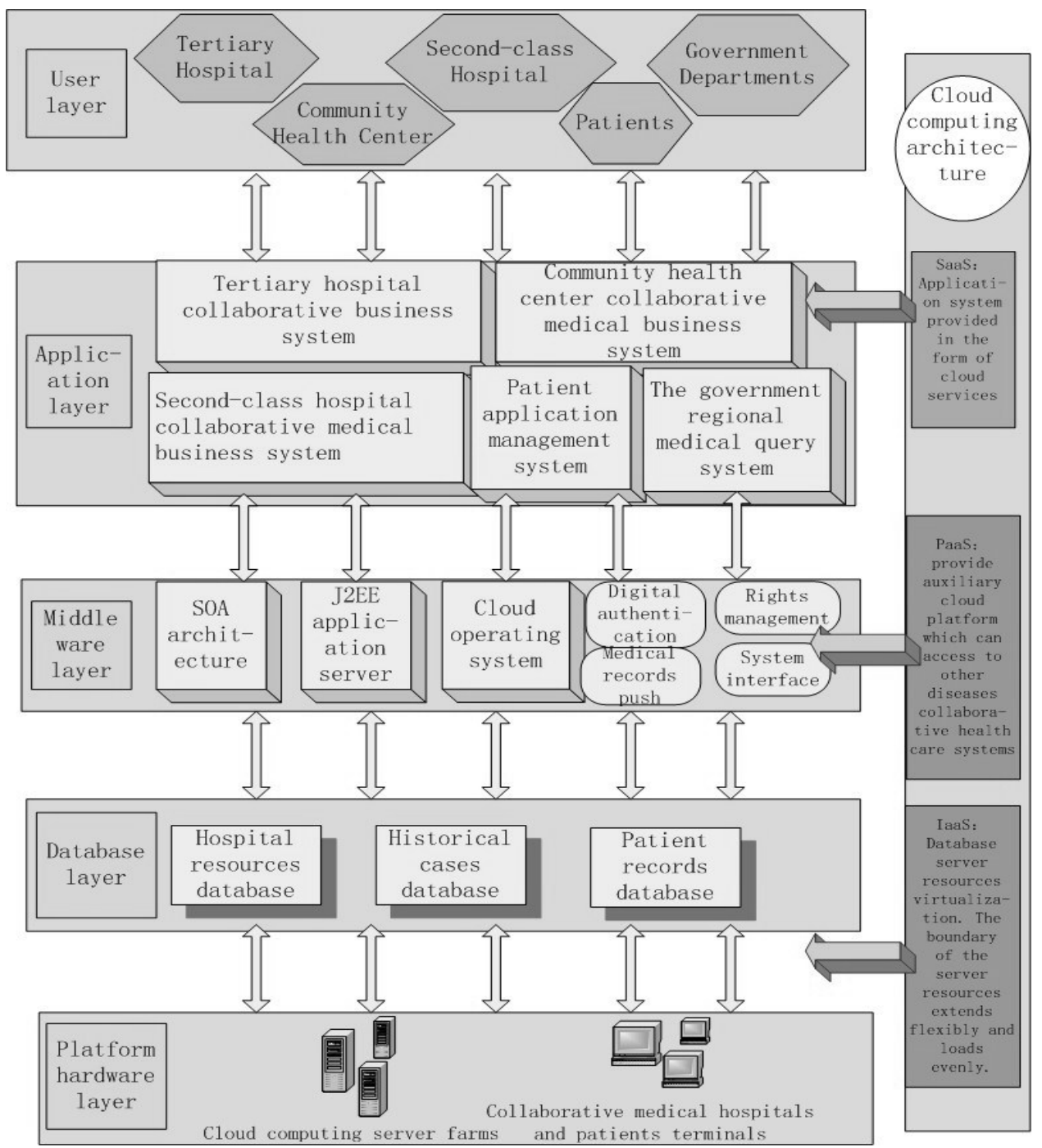

Fig. 3. The application architecture of a regional medical collaborative cloud computing platform

At present there are several mainstream virtualization technologies. On X86 platform, there are HperV in Microsoft and ESX in VMware, etc. On a small platform, there are PowerVM in IBM, vPar in HP, Logical Domain in Sun, etc. The virtualization technology can be selected according to the use-cost and hardware types. In [8] L i has investigated that the hosts, storage devices, the network and other hardware equipments can be processed, such as distributed cluster, abstraction and virtualization. They constitute the entire cloud computing platform 
infrastructures. The differences among the hardware resources will be shielded. The unified automated management will be implemented in the unit of a virtual machine, including resource abstraction, resource monitoring, resource deployment and security management, etc. The utilization rate of the resources can be improved. In addition, the management and maintenance staffs can get rid of the heavy management work, for example, they do not need to manage the hardware resources, the operating systems and so on. But they can focus on the maintenance of the virtual machines and business systems, in order to simplify the management and maintenance work of the data centre.

2. Middleware layer. The middleware layer corresponds to the PaaS layer of cloud computing. It can provide an auxiliary cloud platform which can access other diseases cooperative medical systems through digital authentication, unified system interface, etc.

The development of the cloud computing platform can use the Hadoop framework. The cores of Hadoop are a distributed file system, distributed data processing and distributed structured data tables. They can meet the needs of a medical data centre and ensure the high reliability, high availability and scalability. The Hadoop distributed file system has relatively complete redundancy backup and a fault recovery mechanism and can be deployed on cheap hardware. It can also support load balancing strategy to ensure scalability. In addition, the distributed data processing and distributed structured data table can support structured storage, shield the underlying distributed programming to reduce the development difficulty and ensure high throughput access to medical data.

If users want to develop and operate software in the cloud computing platform, the cloud computing platform must provide the corresponding operation environment. The operation environment must satisfy the service definition, service management and service invocation. Service-oriented infrastructure SOA meets all the requirements in a cloud computing service life cycle. SOA infrastructure can convert similar businesses to a set of interrelated services or repeated businesses. When users use these businesses or services, they can be accessed through Internet. SOA architecture can efficiently integrate these services to complete the specific business needs. SOA architecture emphasizes the service first and aligns business and IT. It is good for the medical institutions to respond to the quick change of social needs.

Jboss can be chosen as a J2EE application server. Jboss has many good qualities. It uses JMX micro kernel service as its bus structure and it is of serviceoriented architecture. It has also a unified class load. So it is a highly modular and loosely coupled. When using the J2EE to develop a web application, we can adopt the way of JSP, Servlet and Bean. The JSP is used to implement the view and the servlet is used to control the program part and a bean is used to implement the model part of the program, which is called the MVC pattern, namely model, view and control. Using the MVC pattern can realize the separation of the code. In the MVC pattern, three layers carry out their duties. So when one of these three layers is changed, you only need to change the code corresponding layer without affecting other layers of code. 
3. Application layer and user layer. The application layer and user layer correspond to the SaaS layer of cloud computing. Systems in the application layer can provide services to users. In [9] Y u e, Z h a n g have investigated that the SaaS layer mainly provides support to run the software and enables the users to acquire fast the delivery of software. So they can get more professional software services by investing in less IT resources. In the mode of IT as a service mode, the service providers can provide hardware and software services via Internet. The users can rent the cloud computing services paying related fees and they do not need to manage and maintain the software. In general, the users can use the cloud services through a Web browser, but in order to meet the requirements of complicated medical institutions, custom clients which can provide cloud services can be developed.

The regional medical cloud computing platform helps to break the traditional disadvantages of medical and health informatization construction and can effectively integrate clinical data of patients in different medical institutions information systems and other health information. It can also provide collaborative service platform for the different levels of the medical staff of medical institutions to carry out a collaborative service model based on the timely sharing of the patient's data.

3.3. The problems in the process of developing a regional health collaborative cloud computing platform

When developing a regional medical collaborative cloud computing platform, we need to solve some problems.

1. Heterogeneous data conversion. Data in different medical institutions are heterogeneous, because the hardware platforms, database systems and organization methods of the medical institutions are different and there is a lack of unified planning. We can use XML and BASE64 encoding technology to solve the problem of heterogeneous data format conversion. XML is designed by World Wide Web Consortium (W3C) and has good extensibility. It can flexibly express various text data information

2. Data security. If the cloud centre of the medical data is established, the data security, reliability, and the patients' privacy must be considered. The methods are as follows. The security policies of the architectural layer can use user's authentication and authorization, data isolation and data encryption. The patient data can use anonymity. The privacy of the data on the storage, operation, network transmission must be ensured.

3. Standards formulation. Because medical institutions use separate database systems, the data codes are different. In order to realize unified data encoding, the unified coding standard and data dictionary should be formulated. In addition, a medical cloud storage standard and a business processes standard, and so on must be formulated to ensure the quality of the cloud computing services. 
4. The regional medical business process optimization based on cloud computing

If cloud computing can be used in the health care industry, there will be a hope to optimize the regional medical business process.

\subsection{The regional medical business process optimization}

In [10] $\mathrm{L} \mathrm{i}$ has investigated that business process optimization is a strategy that develops, improves and optimizes the business processes to maintain competitive advantage. Fig. 4 shows the optimized regional medical business process.

\section{Primary hespital}

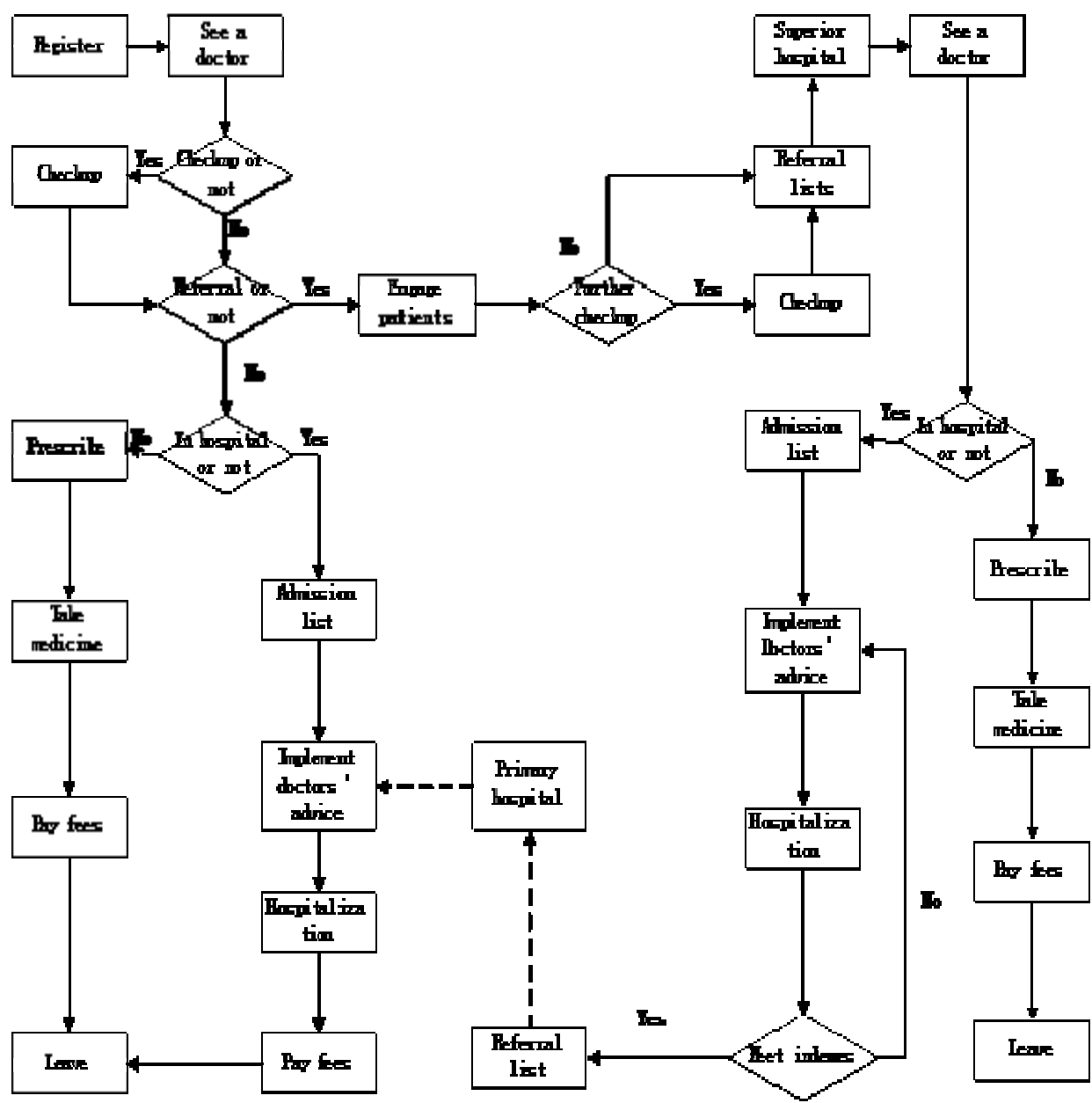

Fig. 4. The optimized regional medical business process 
Diagnosis in community (Primary hospital).The patient should register and pay some money in advance saved in their electronic accounts. Then they take the registration form to wait to see a doctor. The doctor will make the judge whether the patients need to check or not. If they do not need to check, the doctor will prescribe directly. If they need to check, they can have a check directly. After several hours, they get the check results. After seeing the results, the doctor will make the judge whether they have the ability to solve the disease. If they can, they will prescribe and share the patients' information to the cloud computing platform. And then the patients get the medicine directly. If the patients need to be in a hospital, they can have a treatment. When they are recovered, they will pay the fees and leave the primary hospital. Need to explain, that every time when patients have paid fees, the doctor will check the account balance of the patients. If it is enough, the patients can have a check or take the medicine first and pay the fees at last. If it is not enough, the patients need to save some money in their electronic account and then have a check or take a medicine.

Upward referral. If the primary hospital doctors have no ability to solve the disease, they will transfer the patients to the superior hospital. If the patients agree to referral, the doctor will fill out the upward referral list and then the business people will send it to the superior hospital. The doctors in the superior hospital will make a judge whether the patients need ambulatory treatment or hospital care. If they need ambulatory treatment, they do not need to register and check again. The doctor in the superior hospital can directly obtain patients' checkup information in the primary hospital through the cloud computing platform. And then the doctor will prescribe. The patients take the medicine and pay fees. If they need a hospital care, they need to register and will be allocated a bed and an attending doctor. The attending doctor will give doctor's orders according to the check results, including the shared results and new check results. The doctors' orders will be shared in the cloud computing platform. The nurses will execute them. When the condition of the patients is improved after a period of treatment and meets the indicators, the patients can be transferred to the community hospital for rehabilitation.

Downward referral. If the patients agree to be transferred to the community hospital for rehabilitation, the business people will send the downward referral list written by the doctor to the primary hospital. The doctors and nurses in the primary hospital will obtain the superior hospitals' doctors' orders through the cloud computing platform. They will do rehabilitation treatment for the patients. When the patients are recovered, they will pay fees and leave.

\subsection{Analysis of regional medical business process optimization}

Through analysis of the architecture of the regional medical collaborative cloud computing platform based on cloud computing, we know that it can realize sharing of the medical resources. So it can realize regional medical business process optimization.

1. Provide basic information only once. The patients provide their basic information in their first diagnosed registration, and then their basic information is stored in the cloud computing platform. After referral, they do not need to register, 
because the higher hospital can directly access the information through the cloud computing platform.

2. One-time payment. Patients' electronic accounts can be established and shared to all regional medical institutions. If the balance of the patients' electronic account is enough, they can pay in any medical institution only once when the entire checkup is finished.

3. Checkup. Because the patients' checkup information can be shared, including the laboratory sheet, image data and so on, the doctors in the superior hospital can directly obtain their checkup information in the primary hospital through the cloud computing platform to make a further check of the patients.

4. Doctor's orders. By sharing doctor's orders of superior hospitals in the cloud computing platform, the doctors and nurses in the primary hospital can be able to quickly and accurately obtain the doctor's orders about the patients in order to ensure patients' rehabilitation.

5. Shorten the hospitalization period. The checkup information about the patients is shared in the cloud computing platform, so the doctor can quickly propose a treatment by obtaining the information from the cloud computing platform. The time for checking and waiting for results is eliminated. When living in the ward, the patients can receive treatment quickly, so the hospitalization period is shortened.

\section{Summary}

Cloud computing technology has injected new vitality into the development of regional medical informatization and can improve the medical industry. It brings favourable conditions for realizing medical resource sharing among medical institutions. We need to do a further study and design about the regional medical collaborative cloud computing platform. The cloud computing platform is important to expand the coverage of high-quality medical resources, improving traditional medical service mode and optimizing regional medical business process, and so on. However, the process optimization cannot be achieved only once, but it is a continuous reform and a never-ending process. It needs to be constantly improved to make it more scientific and more reasonable. But how to reduce medical care costs through business process optimization and how to distribute profit needs to be studied further. Nevertheless, we believe that in the near future, cloud computing will certainly play a significant role in the work of regional medical informatization and will have a positive and far-reaching impact on the further development of regional medical informatization.

Acknowledgment: The authors would like to express their great thanks to the support from the Beijing municipal science \& technology commission Grant No Z131100005613017. 


\section{References}

1. H a n, J. The Reviews of the Cloud Computing. - The Development of the Company Technology, Vol. 29, 2012, No 15, 13-14.

2. Z h a o, P. Advocate Medical Resources Sharing and Promote the Development of the Hospitals. Health Economic Research, Vol. 30, 2002, No 6, 40-41.

3. La i, Y. C., Z. S ong. The Importance of Business Process Reengineering for the Hospital Informatization Construction. - Chinese Journal of Hospital Management, Vol. 20, 2004, No $15,750-752$.

4. H o w a r d, S., F. P e t e r. Business Process Management: the Third Wave. - Meghan Kiffer Press, Vol. 10, 2002, No 6, 80-110.

5. W a n g, S., W. S u. The Development Status, Existing Problems and the Countermeasure Study of Our Country's Regional Health Informatization. - Modern Preventive Medicine, Vol. 37, 2010, No 22, 4241-4243.

6. T a n, C., K. L i u, L. S u n. A Design of Evaluation Method for SaaS in Cloud Computing. Journal of Industrial Engineering and Management, Vol. 6, 2013, No 1, 50-72.

7. Y a n, G. Cloud Computing in the Application of Regional Health Informatization. Beijing, Beijing Jiaotong University, 2011, 15-30.

8. L i, Q. Introduction to the Applications of the Cloud Computing. - Dossier, Vol. 28, 2012, No 2, 104-105.

9. Y u e, H. L., K. Z h a n g. Cloud Computing in the Application of Regional Health Informatization. - Computer and Modernization, Vol. 27, 2012, No 8, 141-143.

10. L i, R. Study on Mobile e-Commerce Business Process Optimization. - Journal of System and Management Sciences, Vol. 2, 2012, No 2, 46-53. 